\title{
Investigations on tungsten heavy alloys for use as plasma facing material
}

\author{
R. Neu ${ }^{\mathrm{a}, \mathrm{b}}$, H. Maier ${ }^{\mathrm{a}}$, M. Balden ${ }^{\mathrm{a}}$, S. Elgetia ${ }^{\mathrm{a}}$, H. Gietl ${ }^{\mathrm{a}, \mathrm{b}}$, H. Greuner $^{\mathrm{a}}$, A. Herrmann ${ }^{\mathrm{a}}$, A. Houben ${ }^{\mathrm{c}}$, V. Rohde ${ }^{\mathrm{a}}$, B. Sieglin ${ }^{\mathrm{a}}$, \\ I. Zammuto ${ }^{\mathrm{a}}$, ASDEX Upgrade Team ${ }^{\mathrm{a}}$ \\ ${ }^{a}$ Max-Planck-Institut für Plasmaphysik, 85748 Garching, Germany \\ ${ }^{b}$ Technische Universität München, Boltzmannstrasse 15, 85748 Garching, Germany \\ ${ }^{c}$ Forschungszentrum Jülich GmbH, Institut für Energie und Klimaforschung - Plasmaphysik, Partner of the Trilateral Euregio Cluster, 52425 Jülich, Germany
}

\begin{abstract}
An alternative solution for tungsten as a plasma facing material could be the use of $\mathrm{W}$ heavy alloys as they are produced commercially by several companies. They consist of up to $97 \% \mathrm{~W}$ and $\mathrm{Ni} / \mathrm{Fe}$ (or Ni/Cu) admixtures, they are readily machinable and considerably cheaper than bulk tungsten. Their major drawbacks in view of the application in fusion experiments are the rather low melting temperature and their magnetic properties (in case of a Ni/Fe binder phase). In a first step $\mathrm{W}$ heavy alloys from two manufacturers were investigated concerning their thermal and magnetic properties and subjected to screening tests and cyclic loading in the high heat flux test facility GLADIS with up to $20 \mathrm{MWm}^{-2}$ and surface temperatures of up to $2200^{\circ} \mathrm{C}$, showing no macroscopic failure. SEM investigations show a segregation of $\mathrm{Ni}$ and $\mathrm{Fe}$ at the top surface after the thermal overloading, but no signs of microcracking. The long-term behaviour of a W-Ni/Fe tile under plasma and electromagnetic load was investigated in ASDEX Upgrade using its divertor manipulator. The tile was exposed in discharges with record values of injected energy and power. Despite the observed surface modifications ( $\mathrm{Ni} / \mathrm{Fe}$ segregation) the $\mathrm{W}$ heavy alloys seem to provide a pragmatic and cost-effective alternative to bulk $\mathrm{W}$ tiles in the divertor of ASDEX Upgrade.
\end{abstract}

Keywords: tungsten heavy alloy, tungsten, plasma-facing components, high heat flux testing, ASDEX Upgrade

\section{Introduction}

Since 2014 ASDEX Upgrade (AUG) is using bulk tungsten (W) tiles at the outer divertor strike-point. In two experimental campaigns more than 2000 plasma discharges with up to 10 s duration and $100 \mathrm{MJ}$ plasma heating were successfully conducted, without impairment by the $\mathrm{W}$ tiles. However, inspections after the campaigns revealed that a large number of tiles suffered from deep cracking attributed to brittle fracture [1]. A possible option at least for non-nuclear fusion devices could be the use of more ductile $\mathrm{W}$ heavy alloys as they are produced commercially by several companies. It consists of up to $97 \%$ (weight) $\mathrm{W}$ and $\mathrm{Ni} / \mathrm{Fe}$ (or $\mathrm{Ni} / \mathrm{Cu}$ ) admixtures. According to $[2,3]$, the endurance limits of the $\mathrm{W}$ heavy alloys should be considerably larger than that of W (see for example [4]) which should help to avoid cracks under the cyclic load. Their major drawbacks, in view of the application in fusion experiments, are the rather low melting temperature $\left(\approx 1500{ }^{\circ} \mathrm{C}\right.$ or $<1100{ }^{\circ} \mathrm{C}$, respectively) and the higher vapour pressure of the binder phase and their magnetic properties (in the case of $\mathrm{Ni} / \mathrm{Fe}$ admixture).

In order to explore their feasibility, W heavy alloys from two suppliers (Plansee Composite Materials GmbH and HC Starck Hermsdorf $\mathrm{GmbH}$ ) were investigated concerning their thermal and magnetic properties and subjected to screening tests and cyclic loading in the high heat flux test facility GLADIS. In order to gain first experience under cyclic plasma load and to compare its behaviour to the divertor tiles made of bulk tungsten, one tungsten heavy alloy tile was exposed with the divertor manipulator in high power AUG discharges.

\begin{tabular}{|c|c|c|c|c|c|c|}
\hline Name & Supplier & $\begin{array}{c}\text { W } \\
\text { wt.\% }\end{array}$ & $\begin{array}{c}\text { Ni } \\
\text { wt.\% }\end{array}$ & $\begin{array}{c}\text { Fe } \\
\text { wt.\% }\end{array}$ & $\begin{array}{c}\mathrm{Cu} \\
\text { wt.\% }\end{array}$ & $\begin{array}{c}\text { Density } \\
\mathrm{g} \mathrm{cm}^{-3}\end{array}$ \\
\hline D185 & Plansee & 97 & 2.0 & 1.0 & 0.0 & 18.5 \\
HPM1850 & HC Starck & 97 & 2.0 & 1.0 & 0.0 & 18.5 \\
IT180 & Plansee & 95 & 3.5 & 0.0 & 1.5 & 18.0 \\
HPM1801 & HC Starck & 95 & 3.5 & 0.0 & 1.5 & 18.0 \\
\hline
\end{tabular}

Table 1: Names, suppliers, composition and density of the tungsten heavy alloys under investigation.

In Sec. 2 the measured properties (magnetic, thermal and surface composition) of the investigated materials will be presented. Sec. 3 presents the results of high heat flux screening and cycling loading including metallographic investigations, whereas Sec. 4 reports on the behaviour observed in divertor of AUG. Sec. 5 concludes the paper and provides the outlook on further activities.

\section{Properties of Tungsten Heavy Alloys}

Tungsten heavy alloys are compounds with a large fraction of tungsten and admixtures of either $\mathrm{Ni} / \mathrm{Fe}$ or $\mathrm{Ni} / \mathrm{Cu}$ produced by pressing and sintering of powders or liquid phase sintering. Several commercial suppliers offer various grades of these $\mathrm{W}$ heavy alloys and they are widely used as shielding material and balancing weight because of the high atomic number (shielding) and high density (shielding, balancing). Table 1 summarizes the names, suppliers, composition and density of the tungsten heavy alloys under investigation. In respect to tungsten these alloys are considerably cheaper due to the facilitated 
sintering process and they show improved machinability and ductility at room temperature. The latter points could provide essential advantages when being used as plasma facing material in present day fusion devices because of the many thermal cycles they are undergoing due to the pulsed operation and the cyclic loading through ELMs. The downside of the material is, of course, the low melting point of the alloying elements $\mathrm{Fe}$, $\mathrm{Ni}$ and $\mathrm{Cu}$ being $1538{ }^{\circ} \mathrm{C}, 1455^{\circ} \mathrm{C}$ and $1085^{\circ} \mathrm{C}$, respectively, which considerable restricts the operational space. However, when trying to avoid recrystallisation of tungsten to preserve its original mechanical properties the temperature should be kept anyway below $1300{ }^{\circ} \mathrm{C}$, which means that the use of $\mathrm{Fe}$ and $\mathrm{Ni}$ should not affect the operation too strongly. Other issues to be taken into account when using $\mathrm{W}$ heavy alloys are their magnetic properties and their thermal conductivity. Fig. 1 shows a

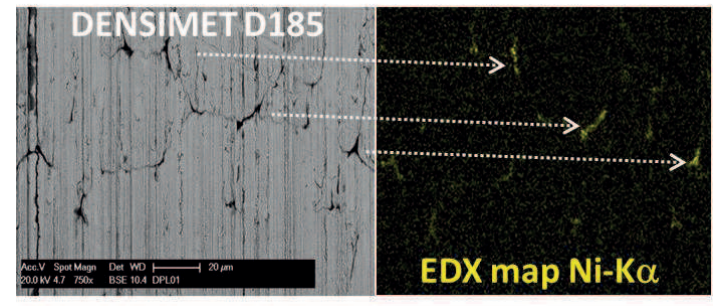

Figure 1: SEM image and EDX map of the DENSIMET D185 surface (machined, as received).

typical scanning electron (SEM) image of a DENSIMET D185 surface in its initial (as machined) state. Nickel (as well as iron) can be identified at the boundaries of large grains as demonstrated on the right side of the figure which is obtained by mapping of the characteristic X-ray emission by energy dispersive $\mathrm{x}$-ray spectroscopy (EDX).

As stated above, it is important to know the detailed magnetic properties when using materials inside a fusion device because of the potential perturbation of the local magnetic field as well as the forces and moments the tiles will experience. Therefore the magnetisation was measured up to a magnetic field of $3 \cdot 10^{4}$ Oe ( $\simeq 3 \mathrm{~T}$ vacuum field) at room temperature (see top of Fig. 2) for D185 and HPM1850 and at temperatures up to $530{ }^{\circ} \mathrm{C}$ (HPM1850 only, Figs. 2 and 3). The magnetisation saturates very quickly with increasing magnetic field at very moderate levels (the magnetisation of Eurofer, which is also tested in AUG (see [5]) is a hundred times larger).

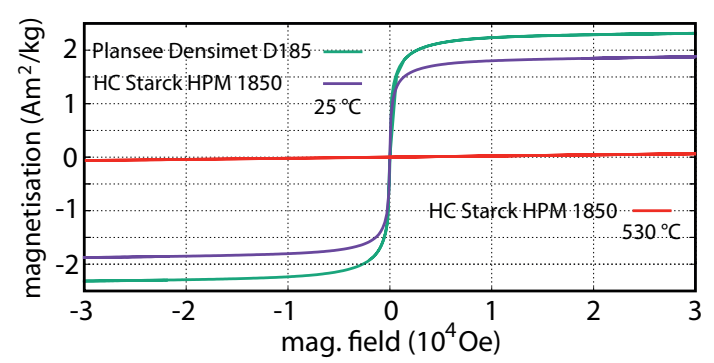

Figure 2: Magnetisation of HPM1850 and D185 as a function of magnetic field at $25^{\circ} \mathrm{C}$ and $530{ }^{\circ} \mathrm{C}$ (HPM1850 only).

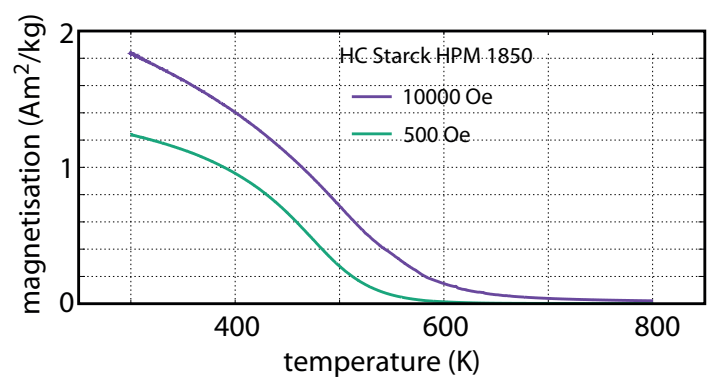

Figure 3: Magnetisation of HPM1850 as a function of temperature.

The magnetisation of D185 is about $20 \%$ higher than that of HPM1850, which might be explained by a slightly higher Fe content in D185 (the specification comprises $1.0 \pm 0.3$ weight $\% \mathrm{Fe}$ ). HPM1850 loses its ferromagnetism above $\mathrm{T} \approx 330^{\circ} \mathrm{C}$ which hints to the fact that in this case the magnetic properties are dominated by $\mathrm{Ni}$.

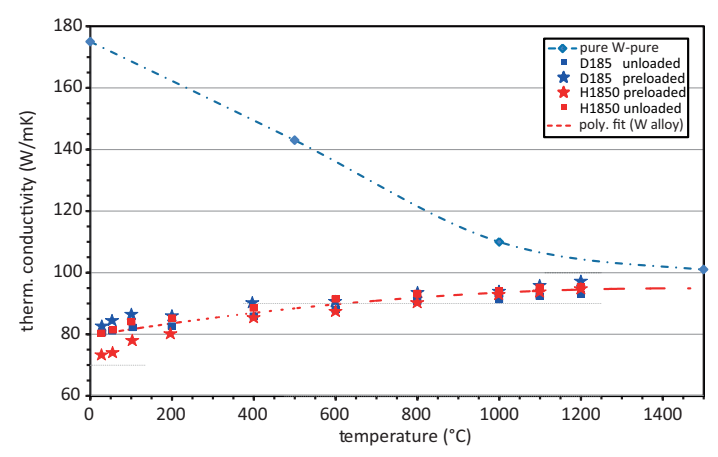

Figure 4: Thermal conductivity of HPM1850, D185 and pure W (data taken from [6]) as a function of temperature. Within the errors of the measurement there is no change of the thermal conductivity in case of previous thermal overloading (see Sec. 3).

The thermal diffusivity was measured by the laser flash method using a laser flash apparatus (NETZSCH, LFA 427) in the range from $50{ }^{\circ} \mathrm{C}$ up to $1200{ }^{\circ} \mathrm{C}$ (laser: $\mathrm{Nd}$ :Cr:GGG, wave length $1064 \mathrm{~nm}$, detector: InSb, atmosphere: Ar flux 190 $\mathrm{ml} / \mathrm{min}$ ). The thermal conductivity was determined by multiplying the thermal diffusivity with density and specific heat. The temperature dependent data of specific heat of pure $\mathrm{Fe}, \mathrm{Ni}$, and $\mathrm{W}$ are taken from [7] combining them linearly according to their fractional mass. The density $\left(18.5 \mathrm{gcm}^{-3}\right)$ was assumed to be approximately constant. The thermal conductivity of D185 and HPM1850 is very similar, irrespectively whether the material has been overloaded (see Sec. 3) or it is in its initial state (the differences in the measurements at a certain temperature are within the estimated error). Interestingly the thermal conductivity slightly increased from about $80 \mathrm{~W}(\mathrm{mK})^{-1}$ at $50{ }^{\circ} \mathrm{C}$ up to $95 \mathrm{~W}(\mathrm{mK})^{-1}$ at $1200{ }^{\circ} \mathrm{C}$. As a consequence, the thermal response of the surface of a HHF loaded component is very similar as for bulk tungsten although at room temperature the thermal conductivity of $\mathrm{W}$ is almost twice see also Sec. 3. 


\section{High Heat Flux Tests in GLADIS}

In order to characterise the behaviour of tungsten heavy alloys under high heat fluxes (HHF), tiles with identical geometry as the AUG divertor components were exposed in the high heat flux test facility GLADIS [8]. During these adiabatical loading tests the tiles are exposed to a hydrogen beam with a gaussian shape of $75 \mathrm{~mm}$ FWHM and a maximum central power density of $20 \mathrm{MWm}^{-2}$. Since the tiles have the dimensions of $227 \times 77 \times 15 \mathrm{~mm}^{3}$, two thermally independent test series per tile could be performed by changing the test position accordingly.

Besides the two W-Ni/Fe heavy alloys (D185, HP 1850), INERMET IT 180 (Plansee) and HPM1801 (HC Starck), both consisting of $\mathrm{W}(95 \%), \mathrm{Ni}(3.5 \%)$ and $\mathrm{Cu}(1.5 \%)$ (all weight $\%$ ), were also tested as a risk mitigation alternative in case the magnetisation of the $\mathrm{W}-\mathrm{Ni} / \mathrm{Fe}$ heavy alloys would have been too high (the HHF tests were done in parallel to the other investigations). The performed tests consisted of two parts:

- thermal screening where the deposited energy and power was increased up to damage of the material in order to find the limit for the acceptable load and to investigate the material's behaviour under overload, and

- thermal cycling using a moderate power/energy load, similar to that expected in AUG in order to investigate the low cycle fatigue.

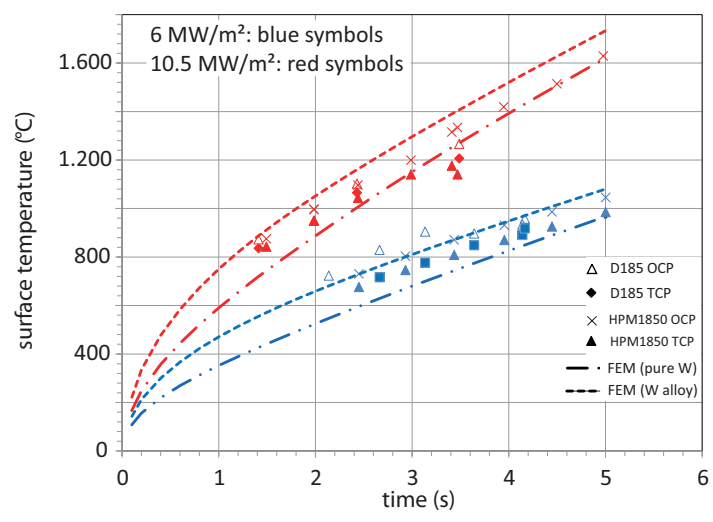

Figure 5: Measured surface temperatures at the end of the loading at 6.0 $\mathrm{MWm}^{-2}$ (blue symbols) and $10.5 \mathrm{MWm}^{-2}$ (red symbols). OCP/TCP: one/two colour pyrometer measurements. The lines result from FEM calculations using the measured thermal conductivity of the W heavy alloy (short dashed lines) und pure W (dash dotted lines) as shown in Fig.4.

Figure 5 shows the measured surface temperatures at the end of the loading at $6.0 \mathrm{MWm}^{-2}$ and $10.5 \mathrm{MWm}^{-2}$ as a function of the pulse duration for D185 and HPM1850. The different symbols represent the two different materials as well as the two different surface temperature measurements with a one and a two colour pyrometer, respectively. In the range where both materials were tested their surface temperature response is very similar. The lines represent surface temperatures simulated by FEM calculations using the measured temperature dependent thermal conductivities of $\mathrm{W}$ heavy alloys (short dashed, see Fig. 4) and of W (dash-dotted) for comparison. The simulations agree rather well in the low temperature range of the 6.0
$\mathrm{MWm}^{-2}$ case, but they slightly overestimate the surface temperature for the longer $10.5 \mathrm{MWm}^{-2}$ loading cases. This reflects the behaviour of the thermal conductivities as shown in Fig. 4. At lower temperatures the $\mathrm{W}-\mathrm{Ni} / \mathrm{Fe}$ heavy alloys perform worse than W (i.e. their surface temperature is higher) whereas at higher temperature the thermal response is very similar to that of W. In fact the observed surface temperatures for 4 - $5 \mathrm{~s}$ loading even suggest that the thermal conductivity at $1400-1600^{\circ} \mathrm{C}$ is as high as the one of W. First surface modifications were observed in the visible and the infrared emission after $3.2 \mathrm{~s}$ at $10.5 \mathrm{MWm}^{-2}\left(T_{\text {surf }} \approx 1300{ }^{\circ} \mathrm{C}\right.$; irradiation conditions exceeding this value will be called 'overload' in the remainder of the paper). The change becomes also obvious when comparing the one and two colour pyrometer signals of HPM1850 for $t \geq 3.5$ $\mathrm{s}$, which strongly deviate for increasing loading durations. No further pulses with duration larger than $3.5 \mathrm{~s}$ where performed for D185 because of the very similar behaviour of both materials (D185 and HPM1850). In addition to the pulses presented in Fig. 5, the HPM1850 tile was further loaded up $20 \mathrm{MW} / \mathrm{m}^{-2}$ for $3 \mathrm{~s}$ as part of screening tests, reaching surface temperatures of up to $2200{ }^{\circ} \mathrm{C}$ in the center of the loading spot. Inspecting the tile after the different pulses it turned out that the initially glossy surface had tarnished. The affected area stayed unchanged for constant loading conditions, but it increased when increasing the load further, reflecting the growing area with $T_{\text {surf }}>1300$ ${ }^{\circ} \mathrm{C}$. Figure 6 shows the surface of the HPM1850 tile at a similar magnification as Fig. 1 after the heavy overload described above. Obviously the $\mathrm{Ni} / \mathrm{Fe}$ has segregated at the surface into larger pools thereby changing the visual appearance. In order
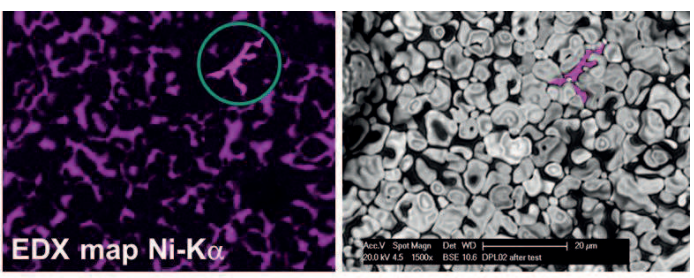

Figure 6: SEM image and EDX map of the HPM1850 tile surface after the high heat flux test in GLADIS with surface temperatures $\leq 2200{ }^{\circ} \mathrm{C}\left(20 \mathrm{MW} / \mathrm{m}^{-2}\right.$, $3 \mathrm{~s})$.

to investigate whether this surface segregation could have an influence on the thermo-mechanical behaviour of the material, metallographic cross sections have been produced from the material in its initial state and after the strong overload. As can be judged from Fig.7 only the top few $\mu \mathrm{m}$ (barely visible at this magnification) have changed their structure whereas in the bulk of the material no change is found. The porosity seen on the micrograph of the loaded area is common to all of the material and is also found in unloaded areas. Together with the result on the thermal conductivity presented in Sec. 2 one can expect that the bulk properties do not change under the conditions expected in AUG.

As stated above, similar HHF tests were performed for the $\mathrm{W}-\mathrm{Ni} / \mathrm{Cu}$ heavy alloys IT180 and HPM1801. For moderate thermal loads the observed surface temperatures were lower than the ones for D185 and HPM1850 under similar conditions, 
most probably due to a higher thermal conductivity because of the $\mathrm{Cu}$ content. However, already after $3.3 \mathrm{~s}$ at $10.5 \mathrm{MWm}^{-2}$ $\left(\approx 1050{ }^{\circ} \mathrm{C}\right.$ ) a violent ejection of $\mathrm{Cu}$ is observed during the GLADIS thermal screening tests, leaving large craters (several hundred of micrometers) on the tile surfaces. The reason for this strongly different behaviour is not clear, but it might be attributed to the larger vapour pressure of $\mathrm{Cu}$ already at $1050{ }^{\circ} \mathrm{C}$, which is about two orders of magnitude higher than that of $\mathrm{Fe}$. Such a catastrophic failure and the strong surface modification under overload could hamper the plasma discharges in AUG and therefore the use of such tiles as PFCs in the divertor was discarded.
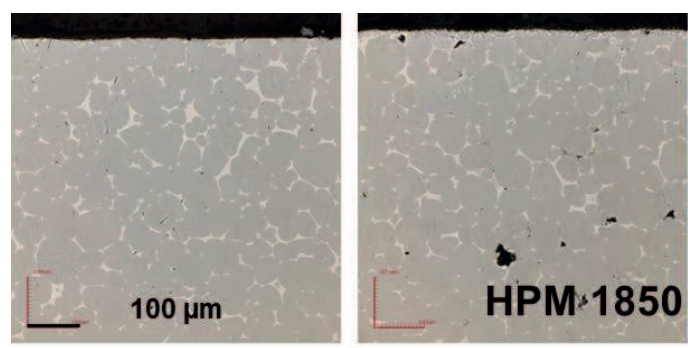

Figure 7: Metallographic cross section of the HPM1850 tile in its initial state and after strong overload (cf. Fig. 6).

The second part of the HHF flux tests for D185 and HP 1850 , namely the cyclic loading was performed with 50 pulses at 10.5 $\mathrm{MWm}^{-2}$ with a pulse length of $2.5 \mathrm{~s}$, leading to typical surface temperatures in the range $1050-1100{ }^{\circ} \mathrm{C}$ ). According to routine infrared measurements in the divertor of AUG most of the discharges stay well below this temperature, making these test conditions a relevant approach. During this pulse cycle $T_{\text {surf }}$ was continuously monitored and no temporal evolution was observed. Further analyses after the cycling revealed no surface damage and no cracking, providing enough confidence to test the tiles in AUG.

\section{Exposure in the ASDEX Upgrade Divertor}

After the encouraging results of the HHF test in GLADIS one D185 tile was mounted in AUG using the divertor manipulator (DIM II) [9]. This unique device allows the exposure of two complete tiles (which could be elaborately instrumented or even actively heated/cooled) for single experimental days or for long experimental periods. Before mounting, the maximum forces originating from the magnetisation of the tile were calculated. Since saturation is reached already at very small magnetic field the resulting (static magnetic) forces are very moderate. The resulting radial force per tile is in the range of several tens of $\mathrm{N}$ and the vertical force is even lower, being negligible compared to the expected electro-magnetic forces during disruptions (which are similar for $\mathrm{W}$ and $\mathrm{W}-\mathrm{Ni} / \mathrm{Fe}$ ). The D185 tile was mounted together with a bulk $\mathrm{W}$ tile for two periods with several weeks of plasma operation. In total they were exposed to 345 plasma discharges with $3 \mathrm{GJ}$ deposited in the outer divertor. The tiles have experienced the discharges with the highest heating power (26 MW) and the highest input energy (> $100 \mathrm{MJ}$ injected up to date into AUG and the maximum power density reached $\approx 17 \mathrm{MWm}^{-2}$ (during the second period). After the first exposure period no cracks (neither D185 nor W) and no surface damage of D185 was found during a visual inspection. However, finally cracks developed on the $\mathrm{W}$ tile very similar to those observed after the previous experimental campaign (see [1]) and segregation of $\mathrm{Ni}$ and Fe was observed on the D185 tile as in GLADIS tests under overload. Figure 8 shows the

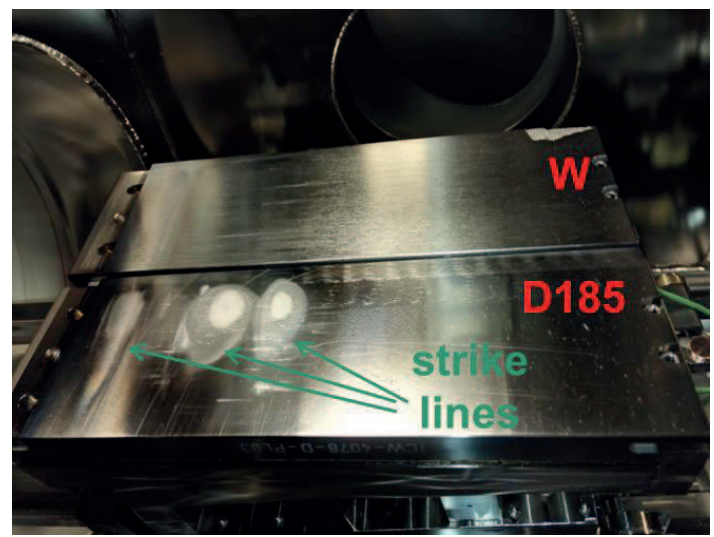

Figure 8: View on the D185 tile and the bulk W tile after exposure with the AUG divertor manipulator.

D185 and the W tiles after exposure in AUG still mounted on the divertor manipulator. The dull areas on the D185 tile mark the different strike line positions and resemble the overloaded areas in the GLADIS tests. Due to tile shadowing by tilting and due to the toroidal geometry in the divertor only parts of the strike line reach the overloading conditions. Despite the obvious surface modification of the D185 tile no adverse effect was observed in AUG. Together with the fact that the tile was exposed to uppermost corners of the operational area of AUG, it has been concluded that the use of $\mathrm{W}-\mathrm{Ni} / \mathrm{Fe}$ heavy alloys could be an option for the use in the divertor of AUG.

\section{Conclusions and Outlook}

Tungsten heavy alloys or tungsten heavy alloys are compounds with a large fraction of tungsten and admixtures of either $\mathrm{Ni} / \mathrm{Fe}$ or $\mathrm{Ni} / \mathrm{Cu}$. In respect to bulk $\mathrm{W}$, these materials are considerably cheaper due to the facilitated sintering process and they show improved machinability and ductility at room temperature. The latter could be beneficial when being used in PFCs because of the thermal cycles due to the pulsed operation and the cyclic loading through ELMs. The low melting point of the alloying elements $\mathrm{Fe}, \mathrm{Ni}$ and $\mathrm{Cu}$ could strongly restrict the operational space depending on the materials behaviour under overload.

In this contribution the temperature dependent magnetisation and thermal conductivities of the commercially available W$\mathrm{Ni} / \mathrm{Fe}$ heavy alloys (D185, Plansee and HPM1850, HC Starck) have been measured. The magnetisation of these materials is moderate $\left(\approx 2 \mathrm{Am}^{-2} \mathrm{~kg}^{-1}\right)$ and saturates already at very low magnetic field. The thermal conductivity at room temperature 
is a factor of two smaller than that of $\mathrm{W}$, but in contrast to $\mathrm{W}$ it rather increases with $T$ leading to a similar thermal performance above $700{ }^{\circ} \mathrm{C}$. The materials were tested under high heat fluxes in GLADIS with power loads of up to $20 \mathrm{MWm}^{-2}$ and surface temperatures of up to $2200{ }^{\circ} \mathrm{C}$. As expected, a surface modification is observed when the melting temperature of the binder phase is exceeded. However in the case of $\mathrm{W}-\mathrm{Ni} / \mathrm{Fe}$ the behaviour under overload is very benign: segregation of $\mathrm{Ni} / \mathrm{Fe}$ in the topmost micrometers but the bulk structure seems not to be affected under the conditions tested here. In contrast, W$\mathrm{Ni} / \mathrm{Cu}$ heavy alloys violently eject $\mathrm{Cu}$ when its melting temperature is exceeded leading to macroscopic surface modifications and possibly unacceptable impurity injection into the plasma. Long term exposure of a D185 tile in AUG under the highest possible power and energy injection confirm the rather positive results of the GLADIS HHF tests. It was therefore decided to equip the divertor of AUG with $26 \mathrm{~W}-\mathrm{Ni} / \mathrm{Fe}$ (HPM1850) tiles, representing more than one fifth of the total divertor (see Fig. 9 ). The rest of the divertor will be equipped with castellated and bisected $\mathrm{W}$ tiles in order to minimize the risk of cracking as observed with the present design. The more ductile $\mathrm{W}-\mathrm{Ni} / \mathrm{Fe}$ tiles will be preferentially mounted at the edges of a sector because there the forces during disruption are expected to be highest. Depending on the performance of the tiles during the upcoming campaign a higher fraction of $\mathrm{W}$ heavy alloy tiles may be installed in the future. A crucial question to be assessed is the microscopic and macroscopic erosion behaviour. Under normal operation conditions the influx of $\mathrm{Ni}$ and $\mathrm{Fe}$ is expected to be very small because according to their fractional abundance only a fraction of the (low) erosion yield of $\mathrm{W}$ is expected in steady state operation. However, after strong overloading and the related segregation of the $\mathrm{Ni}$ and Fe, their influx could be temporarily increased. Moreover, repeated excessive overload could lead to the loss of W grains from the surface. Dedicated spectroscopic monitoring of the impurity influx and content will be performed in order to assess these issues. If the W heavy alloy tiles will allow a stable operation of AUG throughout the next campaigns, they may be used as a pragmatic and costeffective substitute for bulk $\mathrm{W}$ tiles in present day fusion devices. Specifically, when the temperature is kept below 1300 ${ }^{\circ} \mathrm{C}$ to avoid recrystallisation of tungsten to preserve its original mechanical properties, the use of Fe and Ni containing W heavy alloys will not affect the operation excessively.

Whether $\mathrm{W}$ heavy allows can play a role in future fusion reactors (divertor and/or main chamber PFCs) will strongly depend on the foreseen operation conditions and the behaviour of $\mathrm{W}$ heavy alloys under neutron irradiation. Specifically it has to be assessed to what extent nickel could be exchanged with iron as a sintering aid and how the thermo-mechanical properties can be optimized for fusion relevant conditions. Additionally, although it is not an issue in present day devices, the hydrogen retention and the activation behaviour has to be assessed before any conclusion on its suitability can be drawn.

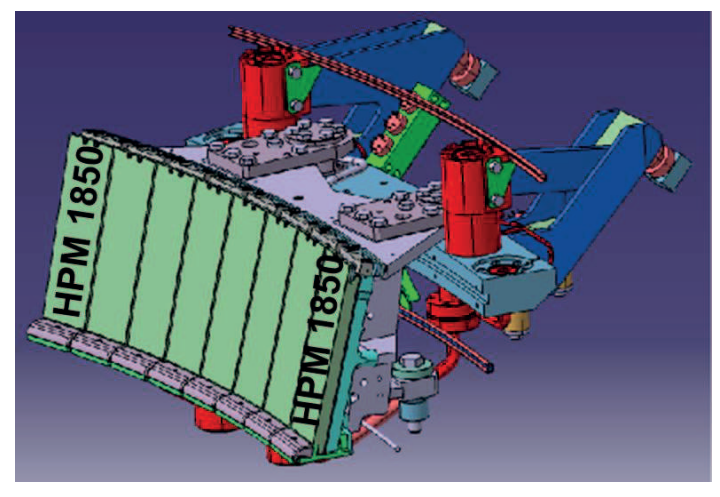

Figure 9: CAD drawing of one sector of the AUG divertor equiped with two HPM1850 tiles.

\section{Acknowledgement}

The authors want to thank M. Li for his help concerning the interpretation of the mechanical fatigue data and B. Böswirth for assisting the GLADIS tests.

\section{References}

[1] A. Herrmann, I. Zammuto, M. Balden, H. Greuner, N. Jaksic, A. Kallenbach, M. Li, R. Neu, V. Rohde, and ASDEX Upgrade team. Experiences with a solid tungsten divertor in ASDEX Upgrade. to be published in $\mathrm{Nu}$ clear Materials and Energy, 2016.

[2] P. Lorenzo, M. Miralda, S. Iyengar, S. Melin, and Etam Noah. International Journal of Refractory Metals and Hard Materials, 41:250 - 258, 2013.

[3] M. Pasalic, F. Rustempasic, S. Iyengar, S. Melin, and E. Noah. International Journal of Refractory Metals and Hard Materials, 42:163 - 168, 2014.

[4] J. Habainy, S. Iyengar, Y. Lee, and Y. Dai. Journal of Nuclear Materials, 465:438 - 447, 2015

[5] I. Zammuto, L. Giannone, A. Houben, A. Herrmann, A. Kallenbach, and ASDEX Upgrade Team. Fusion Engineering and Design, 98-99(0):1419 $-1422,2015$.

[6] Iter material properties handbook. Technical Report Rep. 2237RQ, File Code ITER-AM01-3112, ITER Organisation, 2005.

[7] I. Barin. Thermomechanical data of pure substances. VCH, Weinheim; New York, 3rd edition, 1995.

[8] H. Greuner, B. Boeswirth, J. Boscary, and P. McNeely. Journal of Nuclear Materials, 367370, Part B:1444 - 1448, 2007.

[9] A. Herrmann, N. Jaksic, P. Leitenstern, H. Greuner, K. Krieger, P. de Marné, M. Oberkofler, V. Rohde, G. Schall, and ASDEX Upgrade Team. Fusion Engineering and Design, 98-99(0):1496-1499, 2015. 